\title{
Harmonic balance analysis for semiconductor lasers under large- signal modulation
}

\section{Journal Article}

\section{Author(s):}

Odermatt, Stefan; Witzigmann, Bernd; Schmithuesen, Bernhard

Publication date:

2006-09

Permanent link:

https://doi.org/10.3929/ethz-b-000035458

\section{Rights / license:}

In Copyright - Non-Commercial Use Permitted

Originally published in:

Optical and Quantum Electronics 38(12-14), https://doi.org/10.1007/s11082-006-9009-9 


\title{
Harmonic balance analysis for semiconductor lasers under large-signal modulation
}

\author{
STEFAN ODERMAT T ${ }^{1, *}$, BERND WITZIGMA N N ${ }^{1}$ AND \\ B E R N H A R D SCH M T H Ü S E N ${ }^{2}$ \\ ${ }^{1}$ Integrated Systems Laboratory (ETH Zurich), Gloriastrasse 35, CH-8092 Zurich, Switzerland \\ ${ }^{2}$ Synopsys Switzerland LLC, Affolternstrasse 52, CH-8050 Zurich, Switzerland. \\ (*author for correspondence: E-mail: odermatt@iis.ee.ethz.ch)
}

Received 16 August 2006; accepted 18 September 2006

\begin{abstract}
The dynamic characteristics of an edge-emitting laser under large-signal modulation are analyzed in the frequency domain using a harmonic balance method on device level. The simulations reveal the nonlinearities of the carrier dynamics in the quantum well region which strongly influence the optical power in the higher harmonics.
\end{abstract}

Key words: harmonic balance, semiconductor laser, numerical simulation

\section{Introduction}

The dynamic range of linearity is a critical specification in optoelectronic analog modulation applications. Typical cable television (CATV) applications require the composite second-order distortion not to exceed $-60 \mathrm{dBc}$ (Gorfinkel and Luyri 1995). Even if the lasing power changes linearly with the injection current, intrinsic distortion effects are present and higher harmonics draw power from the fundamental signal. The main physical causes of nonlinearity include carrier leakage, spatial and spectral hole burning, free carrier absorption and carrier-photon resonance effects at high modulation frequencies (Lin et al. 1990; Morthier et al. 1991; Gorfinkel and Luyri 1995; Yu et al. 1996). Unlike previous theoretical work using timestepping or quasi small-signal algorithms (Morthier et al. 1991; Yu et al. 1996), we apply a harmonic balance method (Troyanovsky et al. 2000) which allows us to investigate large-signal effects on device level (in contrast to equivalent circuit models).

\section{Numerical modeling: harmonic balance method}

For the description of the semiconductor laser, a multidimensional electro-opto-thermal model is applied. The model comprises the Poisson equation for the electrostatic potential, continuity equation for electrons, holes 
and the local temperature, wave equations for the optical mode profile and modal photon rate equations to describe the coupling between optical and electro-thermal quantities (Odermatt and Witzigmann 2006; Synopsys 2006). The resulting partial differential device equations are spatially discretized on a unstructured simulation mesh resulting in

$$
\partial_{t} \mathbf{q}(\mathbf{x}(t))+\mathbf{y}(\mathbf{x}(t))-\mathbf{w}(t)=0
$$

where $\mathbf{x}$ denotes the solution variable vector, $\mathbf{w}$ denotes explicit time-dependent sources, whereas $\mathbf{q}$ and $\mathbf{y}$ describe the device behaviour. A direct solution by time integration is too time consuming, since time constants in electro-opto-thermal problems differ by several orders of magnitude: quantum well $(\mathrm{QW})$ carrier scattering times are in the ps range, whereas thermal processes happen on the $\mu \mathrm{s}$ to $\mathrm{ms}$ time scale. Hence, more elaborated frequency-domain methods are better suited to the problem.

In the case of an arbitrary (quasi-)periodic source term $\mathbf{w}$, the so called harmonic balance (HB) method can be applied (Troyanovsky et al. 2000, Schmithüsen et al. 2004). In the HB formulation, both, the source vector $\mathbf{w}=\sum_{k=-K}^{K} \mathbf{W}_{k} \exp \left(i \omega_{k} t\right)$ and the solution vector $\mathbf{x}=\sum_{h=-H}^{H} \mathbf{X}_{h} \exp \left(i \omega_{h} t\right)$ are expanded into Fourier series with $H>K$. A formal Fourier transformation of Equation (1) yields

$$
i \mathbf{\Omega} \mathbf{Q}(\mathbf{X})+\mathbf{Y}(\mathbf{X})-\mathbf{W}=0
$$

where $\mathbf{Q}, \mathbf{Y}$ are the finite Fourier series of $\mathbf{q}(\mathbf{x}(t))$ and $\mathbf{y}(\mathbf{x}(t))$ up to order $H$, respectively. $\boldsymbol{\Omega}$ is the frequency matrix resulting from the time derivative and $\mathbf{W}$ is the finite Fourier series of the source term. Equation (2) forms the nonlinear $\mathrm{HB}$ problem, which has to be solved for $\mathbf{X}$. The major advantage of the $\mathrm{HB}$ formulation is that the laser response of large (quasi-)periodic sources can be analyzed in frequency domain. Quantities such as harmonic distortion and intermodulation distortion are obtained in a straight forward manner. The drawback is the demand for computer resources in terms of memory (Troyanovsky et al. 2000; Schmithüsen et al. 2004) (the HB matrix size corresponds to $(2 H+1) \times(2 H+1)$ the size of the de system with a typical $H=10$ ).

\section{Results}

As a reference example to analyze distortion effects under large-signal modulation, a generic (Al,In)GaAs single QW edge-emitting laser emitting at around $980 \mathrm{~nm}$ is used. The cavity length is $400 \mu \mathrm{m}$ and the mirror reflectivity is 0.3 at both facets. The details about the epitaxial layer structure, 


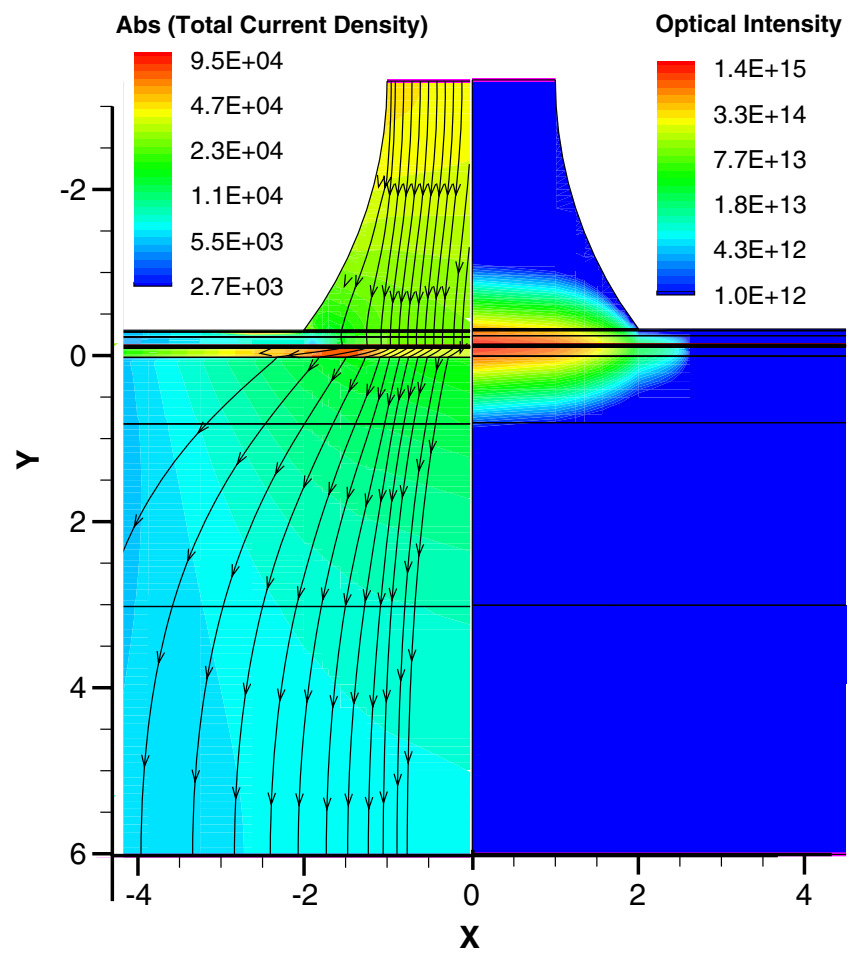

Fig. 1. Static simulation results at bias current $I=100 \mathrm{~mA}$ for the edge-emitting laser described in the text. The current density, profile $\left(\mathrm{A} / \mathrm{cm}^{2}\right)$ is plotted on the left part of the figure. The optical intensity pattern (a.u.) is shown on the right part of the figure.

the simulation setup and cw simulation results are given in Witzig et al. For illustration purposes, Fig. 1 shows the optical mode pattern of the fundamental transverse mode and the current density profile of the 2-D simulation domain at a bias current of $100 \mathrm{~mA}$.

Fig. 2 shows HB simulation results for a bias current of $100 \mathrm{~mA}$ and modulation frequencies of $100 \mathrm{kHz}, 100 \mathrm{MHz}$ and $6 \mathrm{GHz}$ which corresponds to the laser's resonance. With increasing electrical modulation depth, higher order harmonics start to carry more power. Note that the fundamental harmonic increases with $10 \mathrm{~dB} /$ decade, the second harmonic with $20 \mathrm{~dB} /$ decade and the third harmonic with $30 \mathrm{~dB} /$ decade. The nonlinearities are most pronounced for a modulation frequency at the laser's resonance.

The nonlinear distortion effects depend strongly on bias current and modulation frequency. Fig. 3 shows simulation results for varying bias current and modulation frequency. In addition to the increasing resonance frequency of the fundamental harmonic, the higher harmonics also show resonance effects with peak frequencies increasing with bias current which 


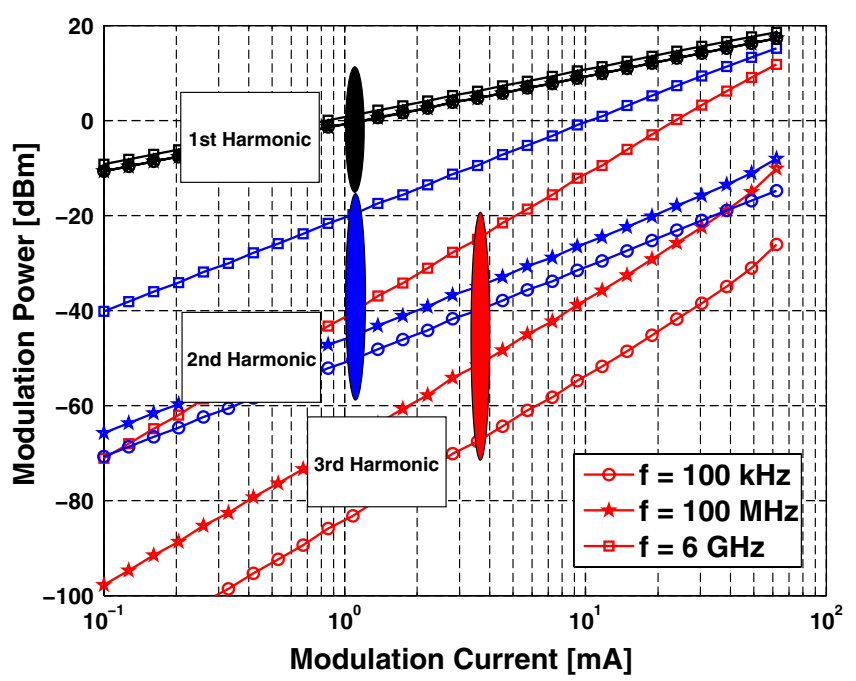

Fig. 2. Harmonic balance simulation results for a bias current of $100 \mathrm{~mA}$ and for different modulation frequencies. With increasing modulation current, the higher order harmonics become stronger. For higher modulation frequencies, the nonlinear effects are more pronounced.

are attributed to stimulated emission processes (Fig. 4): At modulation frequencies below $1 \mathrm{GHz}$, the comparatively fast stimulated emission processes at positions with high optical intensity are able to balance out the harmonic contribution in the carrier densities immediately resulting in very low distortion. Near the resonance frequency of the fundamental harmonic, strong harmonic oscillations are present as well for the carrier density in the QW due to increased interaction. This leads to strong peaks in the second and third order harmonics of the optical output power. At very high frequency well beyond the fundamental resonance frequency, the system cannot follow the excitation any more resulting in decreasing modulation power for all harmonics.

Nonlinearties under modulation are commonly also characterized by second order harmonic distortion (SHD) (Lin et al. 1990, Yu et al. 1996)

$$
\mathrm{SHD}=10 \log \left(\frac{P(2 f)}{P(f)}\right)
$$

with $P(f)$ and $P(2 f)$ being the amplitudes of the first and second harmonic of the optical output power. Fig. 5 shows SHD simulation results for different bias currents and modulation frequencies. In order to obtain low SHD at significantly high frequencies as required by CATV standards (Gorfinkel and Luyri 1995) the laser has to be biased significantly above threshold and the electrical modulation depth has to kept as low as possible. 


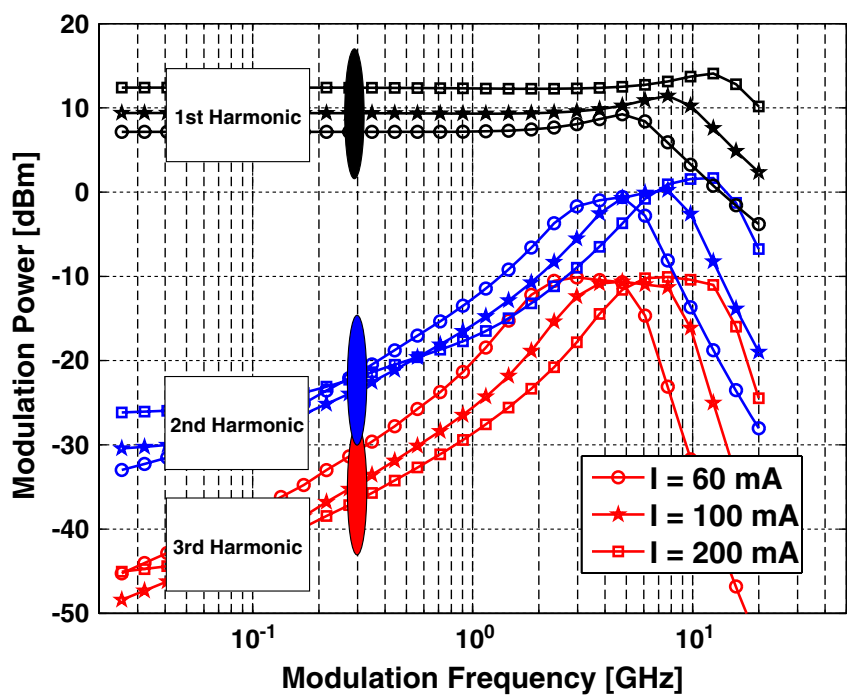

Fig. 3. Frequency-dependent optical modulation power for varying bias current and electrical modulation depth $m=0.1$. The modulation power in the higher harmonics increases with modulation depth and peaks near the resonance frequencies of the harmonics.

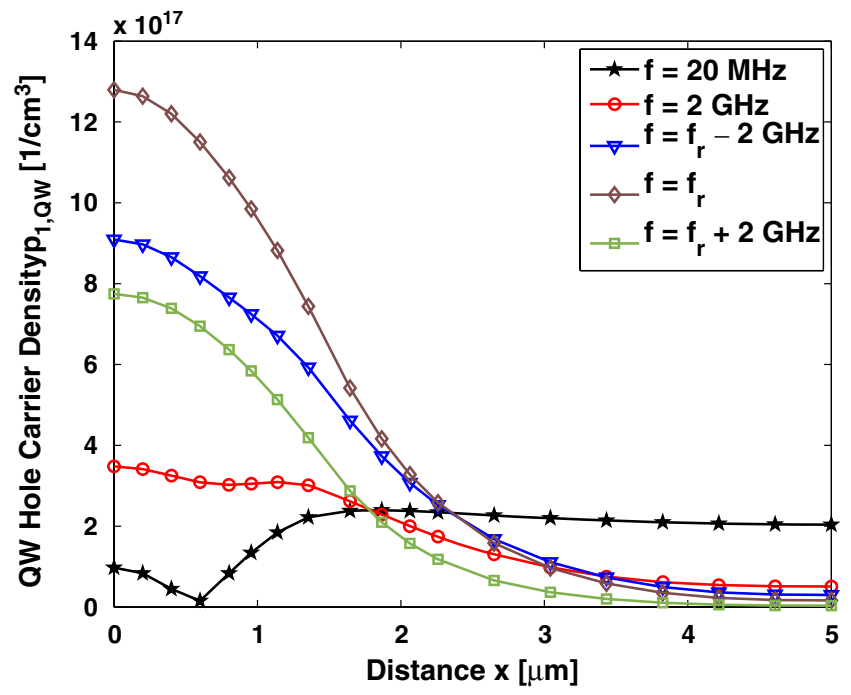

Fig. 4. First harmonic component of the spatially resolved hole carrier density in the quantum well region $p_{1, Q W}$ at bias current $100 \mathrm{~mA}$ and electrical modulation depth $m=0.5$ for different modulation frequencies. Note that the time-independent static contribution $p_{0 . Q W}$ is about $5 \cdot 10^{18} 1 / \mathrm{cm}^{3}$ and that electrons behave similarly. For low frequencies $(<1 \mathrm{GHz})$, fast electron-hole recombination at positions with high optical intensity (i. e. spatial hole burning) reduces $p_{1, Q W}$. At the fundamental resonance frequency $p_{1, Q W}$ peaks at the device center, while it decreases for higher modulation frequencies since the recombination processes are too slow to alter the carrier densities significantly. 


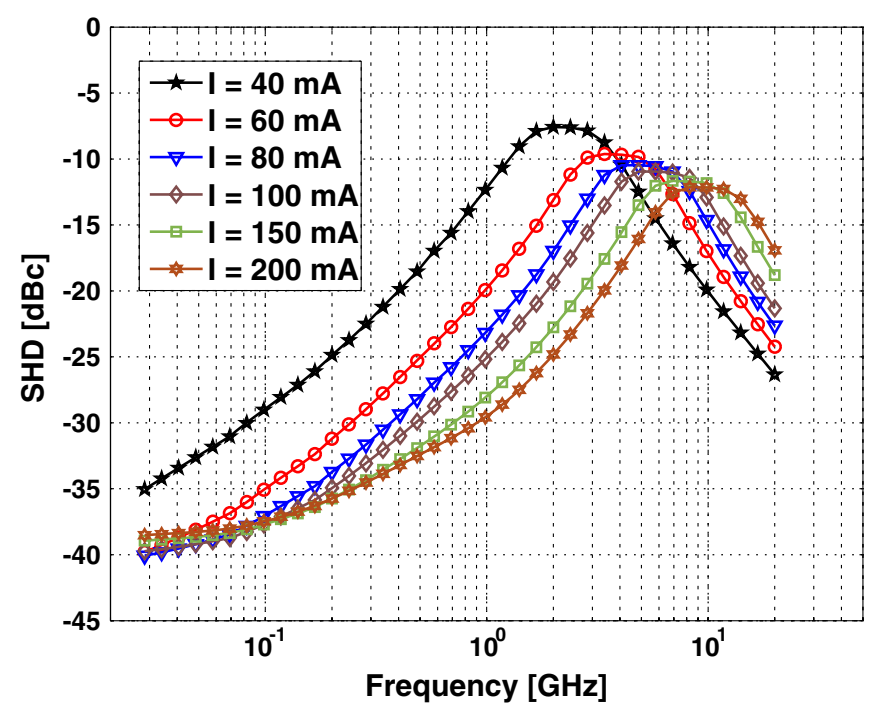

Fig. 5. Frequency-dependent second harmonic distortion for different bias currents at electrical modulation depth $\mathrm{m}=0.1$. While the distortion in the low $\mathrm{GHz}$ regime decreases with increasing bias current due to the shift in the resonance frequency, the distortion is almost bias-independent at very low frequencies $(\ll 100 \mathrm{MHz})$.

\section{Conclusion}

A multi-dimensional large-signal laser model in frequency domain using the harmonic balance method is presented. As example, the nonlinearities in an edge-emitting laser under analog large-signal modulation are analyzed and the detrimental role of oscillating carrier densities for the higher order harmonics is discussed. In general, this frequency domain model is also applicable to digital large-signal modulation.

\section{References}

Gorfinkel, V. and S. Luryi. IEEE. JLT. 13 252, 1995.

Lin, M., S. Wang and N. Dutta. IEEE. JQE. 26 998, 1990.

Morthier, G., F. Libbrecht, K. David, P. Vankwikelberge and R. Baets. IEEE. JQE. 27 1990, 1991.

Yu, S., W. Wong, P. Shum and E. Li. IEEE. JQE. 32, 2139, 1996.

Troyanovsky, B., Z. Yu and R. Dutton. Computational Methods in applied mechanics and engineering 181 467, 2000.

Odermatt, S. and B. Witzigmann. IEEE. JQE. 42 538, 2006.

Synopsys Synopsys Sentaurus Device User Manual 12.0, Mountain View, USA, 2006.

Schmithüsen, B., A. Schenk, I. Ruiz and W. Fichtner. Proc. of Asia Pacific Microwave Conference 483, 2004.

Witzig, A., B. Witzigmann, S. Krishnamurthy, G. Braun, M. Pfeiffer, W. Ng, M. Johnson and W. Fichtner. Proc. SPIE. 5722 454, 2005. 